\title{
Editorial
}

\section{Cell death in yeast: growing applications of a dying buddy}

\author{
D Carmona-Gutierrez ${ }^{1}$, C Ruckenstuhl ${ }^{1}$, MA Bauer ${ }^{1}$, T Eisenberg ${ }^{1}$, S Büttner ${ }^{1}$ and F Madeo ${ }^{\star, 1}$ \\ Cell Death and Differentiation (2010) 17, 733-734. doi:10.1038/cdd.2010.10
}

The discovery of regulated cell death in the budding yeast Saccharomyces cerevisiae and its conservation to the mammalian system has paved the way for a new and fastgrowing research area. ${ }^{1-3}$ Cell death in yeast is often accompanied by diagnostic apoptotic markers such as externalization of phosphatidylserine to the outer leaflet of the plasma membrane, DNA degradation, chromatin condensation, and the generation of reactive oxygen species, all of which can be measured both at a qualitative (microscopic) and at a quantitative (e.g. flow cytometric) level. ${ }^{1} \mathrm{~A}$ teleological explanation for this at first sight counterintuitive phenomenon, in which a unicellular organism succumbs to cellular suicide, has been provided by demonstrating its role in several physiological scenarios, for example, meiosis, aging and mating. ${ }^{4-6}$ Here, the death of damaged individual cells provides a selective advantage for the yeast population as a whole, facilitating the spreading of the clone. In other words, single cells sacrifice themselves for the benefit of the group, which may be regarded as an early root of social biology.

Since the first description of apoptosis in yeast more than a dozen years ago, ${ }^{2,3}$ a growing number of groups have identified crucial molecular regulators of this process, including core executors such as a caspase, ${ }^{7}$ the apoptosis-inducing factor, ${ }^{8}$ endonuclease $\mathrm{G}^{9}$ or the serine protease $\mathrm{OMI},{ }^{10}$ as well as pivotal inhibitors including the AAA-ATPase CDC48 (p97/ $\mathrm{VCP})^{2}$ or the inhibitor of apoptosis protein $B I R 1 .{ }^{11}$ Moreover, yeast cell death has been causally linked to complex apoptotic scenarios such as mitochondrial fragmentation, cytochrome $c$ release, perturbations of the cytoskeleton and epigenetic modifications of the chromatin. ${ }^{1}$ Beyond the study of apoptosis, yeast is currently becoming a valuable tool to investigate other forms of programmed cell death (PCD). In fact, programmed necrosis has recently been described as an important regulatory mechanism during chronological aging in yeast. $^{12}$

The use of yeast as a model organism for cell death research profits from a series of technical benefits such as not being pathogenic, rapid growth, inexpensive accessibility and, most importantly, uncomplicated DNA modification accompanied by simple mutant isolation. Due to the clear-cut, fast and easy genetics, the yeast system allows to establish functional hierarchies and to perform unbiased screenings.
Clonogenic plating assays determining the precise number of dead versus living cells provide a quantitative method to monitor death, which in other organisms is hardly feasible. In addition, the lethal contribution of mitochondria, which, besides being the powerhouses of the cell, are also the main executing organelles upon cell death induction, can be easily explored. This is possible, for instance, by generating cells devoid of mitochondrial DNA (rho ${ }^{0}$ cells) or by enhancing (or suppressing) mitochondrial respiration with a simple transfer into a medium containing a non-fermentable (or fermentable) carbon source. On the other hand, a medium containing oleate as the only carbon source leads to rapid proliferation of peroxisomes and an increase in their size, thus allowing better investigation of the largely unclear impact of these organelles on cell death execution. These advantages illustrate the unique possibility that yeast provides to dissect cell death regulation at a molecular level. In fact, the methodological potential and the wide scope of issues that can be addressed in this system have led to the expansion and diversification of the yeast cell death field into many entirely different directions.

One of these directions has gone towards specifying caspase dependency in different cell death scenarios. ${ }^{13}$ Although caspase-independent pathways have been characterized to involve lethal factors such as the apoptosisinducing factor (AIF1) and endonuclease G (NUC1), to date roughly $40 \%$ of all death conditions tested in yeast have been described to be executed, at least partly, via the yeast metacaspase YCA1. Recently, the phylogenetically conserved protein Tudor staphylococcal nuclease (TSN) was identified as the first biological metacaspase substrate. ${ }^{14}$ At the same time, human TSN, of which cleavage was shown to be important for the progression of apoptosis in mammalian cells, was demonstrated to be a thus far unknown substrate of human caspase-3. ${ }^{14}$ This study establishes that, beyond phylogenetic distance and differences in their proteolytic characteristics, caspases and metacaspases do share natural, death-related target molecules and, hence, do constitute functional homologs.

The identification of cellular components essential for the regulation of apoptosis and clarification of the corresponding mechanisms represent major subjects that are being explored in yeast. For instance, a functional cytoskeleton shows mainly

\footnotetext{
${ }^{1}$ Institute of Molecular Biosciences, Karl-Franzens University, Graz, Austria

${ }^{*}$ Corresponding author: F Madeo, Institute of Molecular Biosciences, Karl-Franzens University, Humboldtstrasse 50/EG, Graz 8010, Austria.

Tel: + 43316380 8878; Fax: + 43316380 9898; E-mail: frank.madeo @uni-graz.at
} 
protective effects against apoptosis in yeast. Actin in particular appears to integrate signals that link nutritional sensing to mitochondria-dependent cell death. ${ }^{15}$ The cell death signalling pathway involving hyperactivation of the Ras protein Ras $2 p$ and activation of PKA (nutrient-dependent protein kinase $A$ ) can be triggered by mutations reducing actin dynamics or enhancing actin aggregation. ${ }^{15}$ Thus, oxidative damage affecting the actin cytoskeleton seems to be carefully monitored by the cell and, if necessary, transformed into an apoptotic response. Given the widespread but mechanistically elusive involvement of actin in apoptosis of diverse eukaryotic organisms, these observations raise the possibility of further exploring the cytoskeleton-apoptotic interconnection, exemplifying the capacity of yeast as an instrument to accomplish pathway finding.

The capability to elucidate cell death-related pathways is further evidenced by the possibility to heterologously express mammalian proteins in yeast that retain functionality but have no direct or obvious sequence orthologs. ${ }^{16}$ These include tumor suppressors such as PTEN or p53, as well as members of the Bcl-2 protein family, allowing for the development of novel approaches that lead to detailed structure-function analyses. In this line, various genes required for Baxmediated cytotoxicity have been identified in yeast. In addition, the yeast system has been used to disclose the specific modulation of apoptosis and $\mathrm{Bcl}-\mathrm{xL}$ phosphorylation by different mammalian protein kinase $\mathrm{C}$ isoforms. ${ }^{17}$ Such analyses, for example, allow the unequivocal characterization of each protein kinase $\mathrm{C}$ isoform, which is more complicated in mammalian cells due to functional overlap and redundancy.

The induction of conserved cell death pathways upon heterologous expression of mammalian proteins has expanded the application of yeast as a model eukaryote to typical 'metazoan-only' topics, such as neurodegeneration. ${ }^{18}$ For example, transgenic yeast expressing alpha-synuclein, the principal toxic trigger during Parkinson's disease, is being used to uncover the underlying mechanisms of this protein's toxicity. ${ }^{19}$ Screens in yeast have yielded important insights into alpha-synuclein's mode of action that have been successfully transferred to Caenorhabditis elegans and mice. The observation that enhancement of vesicular trafficking ameliorates the outcome of the disease in mice, for instance, was based on original results obtained from experiments in yeast. $^{20}$ Thus, yeast has not only become useful to clarify lethal mechanisms upon alpha-synuclein misfolding but also enables the identification of conserved suppressor proteins that are also effectively cytoprotective in mammalian neurons.
The power of yeast apoptosis has further become apparent by directly addressing issues related to cancer biology. These include the analysis of the Warburg effect, a universally occurring metabolic switch from respiratory to fermentative growth in cancer cells. Only recently were the cell survival-promoting effects of suppressed respiration during highly proliferative growth outlined in yeast. ${ }^{21}$ Furthermore, direct medical applicability has emerged from the discovery of apoptotic phenomena in $S$. cerevisiae, which has stimulated intense research about PCD in lower pathogenic eukaryotes in general, such as Candida albicans, Aspergillus, Trypanosoma, Leishmania and Plasmodia. ${ }^{1}$ These works are likely to be of cardinal importance, as they reveal a chink in the difficult-to-treat systemic infections by unicellular organisms.

This special issue, dedicated to yeast apoptosis, covers a broad range of the aforementioned topics, which will contribute to the further identification of conserved components of the apoptotic machinery, help to solve contradictory issues in mammalian PCD and shed light on the underlying mechanisms of diverse human diseases. Budding yeast, as it is and has been in many areas of molecular biology, is lively growing to become a preferred research buddy also when it dies.

\section{Conflict of interest}

The authors declare no conflict of interest.

1. Carmona-Gutierrez D et al. Cell Death Differ, 17: 733-734.

2. Madeo F, Frohlich E, Frohlich KU. J Cell Biol 1997; 139: 729-734.

3. Madeo F et al. J Cell Biol 1999; 145: 757-767.

4. Buttner $S$ et al. J Cell Biol 2006: 521-525.

5. Fabrizio $P$ et al. J Cell Biol 2004; 166: 1055-1067.

6. Herker E et al. J Cell Biol 2004; 164: 501-507.

7. Madeo F et al. Mol Cell 2002; 9: 911-917.

8. Wissing S et al. J Cell Biol 2004; 166: 969-974.

9. Buttner S et al. Mol Cell 2007; 25: 233-246.

10. Fahrenkrog B, Sauder U, Aebi U. J Cell Sci 2004; 117 (Pt 1): 115-126.

11. Walter D et al. J Cell Sci 2006; 119 (Pt 9): 1843-1851.

12. Eisenberg T et al. Nat Cell Biol 2009; 11: 1305-1314.

13. Madeo F et al. Biochem Biophys Res Commun 2009; 382: 227-231.

14. Sundstrom JF et al. Nat Cell Biol 2009; 11: 1347-1354.

15. Leadsham JE et al. Cell Death Differ, 17: 754-762.

16. Greenwood M, Ludovico P. Cell Death Differ, 17: 737-745

17. Saraiva L et al. J Cell Sci 2006; 119 (Pt 15): 3171-3181.

18. Braun RJ et al. Trends Biochem Sci 2009; doi: 10.1016/j.tibs.2009.10.005.

19. Franssens V et al. Cell Death Differ, 17: 746-753.

20. Cooper AA et al. Science 2006; 313: 324-328.

21. Ruckenstuhl C et al. PLoS One 2009; 4: e4592. 